\title{
Publisher Correction: Policy sequencing toward decarbonization
}

Jonas Meckling (1), Thomas Sterner and Gernot Wagner (1)

Correction to: Nature Energy https://doi.org/10.1038/s41560-017-0025-8, published online 13 November 2017.

In the PDF version of this Perspective originally published, the asterisk indicating that the author Gernot Wagner is one of the corresponding authors was omitted. This has now been corrected. 